DFTT 06/2010

June, 2010

\title{
A Method for Measuring the Witten Index Using Lattice Simulation
}

\author{
Issaku Kanamori* \\ INFN sezione di Torino, and \\ Dipartimento di Fisica Teorica, Universita di Torino \\ I-10125 Torino, Italy
}

\begin{abstract}
We propose a method to measure the Witten index using lattice simulation. A requirement for the lattice model is that it has at least one exact supersymmetry at finite lattice spacing. We prove the validity of the method in case of the supersymmetric quantum mechanics, where the index is well known.
\end{abstract}

\section{Introduction}

Recent developments on lattice formulations of supersymmetric theories 1 make it possible to perform numerical simulations to study non-perturbative aspects of the theories. The most important feature in these developments is that the action has (at least) one exact fermionic symmetry at finite lattice spacing. This was pioneered in 2 for gauge theories and in [3] for non-gauge theories. It turned out later that this exact symmetry is the scalar part of supersymmetry in terms of the topological twist. The symmetry is strong enough to guarantee an automatic restoration of the remaining part of the supersymmetry without any fine tuning for the 1- or 2-dimensional case. This restoration is manifestly confirmed numerically in 2-dimensional $\mathcal{N}=(2,2)$ super Yang-Mills [4] using a model by F. Sugino [5], which also has one exact supersymmetry at finite lattice spacing. A more ambitious approach which tries to keep all of the supersymmetry was also proposed by D'Adda et.al. in [6] and its Hopf algebraic structure is studied in [7]. A new proposal along this line is found in 8 . However, without introducing non-locality and/or extra structure such as noncommutativity, only a part of supersymmetry out of full supersymmetry can be realized on the lattice; Especially, the equivalence of [6] to other approaches is well studied in [9]. Not only simple spacetime lattice regularization but also regularization in the momentum space 10 sheds some light on how to regularize supersymmetric theories (see also [11, 12]). A different approach based on the large- $N$ limit is proposed in [13. Further developments in the formulation on the lattice are found in [14, 15, 16, 17.

\footnotetext{
*kanamori@to.infn.it

${ }^{1}$ For a recent review, see Ref. 1 .
} 
One of the most interesting non-perturbative aspects of supersymmetry is the spontaneous breaking of the symmetry. Supersymmetry is broken in our current universe and the breaking should be the result of a non-perturbative effect, unless it is broken from the beginning at the tree level. To study the breaking of supersymmetry using lattice simulation, a Hamiltonian approach was used in [18. The present author with H. Suzuki and F. Sugino proposed a method for detecting the spontaneous supersymmetry breaking using lattice simulation in [19, where they measured the vacuum energy. They also pointed out a possible relation between spontaneous supersymmetry breaking and the existence of a sign problem in the simulation, which comes from the phase of the Pfaffian of the fermion bilinear operator.

Another line of application with large- $N$ limit is found in the gauge/gravity duality. In particular, extensive studies of 1-dimensional model for this purpose are found in Refs. 20, 21. The large- $N$ property of 2 -dimensional $\mathcal{N}=(2,2)$ system has been studied with the same motivation 22, although this system has smaller number of supersymmetries.

In this paper, we propose another method of detecting the spontaneous breaking of supersymmetry. We measure the Witten index [23. If the index is not vanishing, there exists at least one supersymmetric vacuum and supersymmetry is not broken. If the index is vanishing, supersymmetry may or may not be broken. Since the Witten index is a partition function with periodic boundary conditions in the time direction 24, 25, it is of crucial importance to determine the normalization factor in simulation. Usually what we obtain in the simulation are expectation values normalized by the partition function, but what we need here is the normalization itself 2 Our idea to determine the factor consists of two ingredients. First, we regard the derivation of the path integral formalism from the operator formalism as a lattice regularization of the time coordinate. This gives a correct normalization of the path integral measure. Second, we measure a suitably chosen quantity, which cancels a distribution functional $e^{-S}$ in the path integral. Because of this cancellation, we can separate the contribution from the normalization factor. We use supersymmetric quantum mechanics (of $\mathcal{N}=2$ Wess-Zumino type) 27] as an arena for checking our method. This system can be formulated on the lattice in various way 3 . There is a formulation which keeps a nilpotent supercharge $Q$ at finite lattice spacing, with which the action is written in a $Q$-exact form [30, 31, 32]. (See also [33].)

In supersymmetric Yang-Mills quantum mechanics with maximal supersymmetry, which would be an interesting application of our method, the index is of crucial importance. This system is a candidate for M-theory [34, and to guarantee a suitable limit which gives supergravity, the index should be one. Related calculations are found in 35, 36, 37. For this system, a numerical treatment in the Fock space is proposed [38, 39, 40, 41, which is also useful to obtain the Witten index as well. To the author's best knowledge, however, only a little is known about the index from direct calculations of the quantum mechanics so far.

In the next section, we describe our idea in detail. Then we check the validity of the measure by analytically calculating the index for supersymmetric quantum mechanics in the free case in section 3 . In section 4 , we numerically calculate the index of the supersymmetric quantum mechanics and demonstrate that our method in fact reproduces the known index.

\footnotetext{
2 An interesting simulation method which allows to determine the normalization and thus the index is proposed [17. on the basis of the Nicolai map. In a 0-dimensional system, Monte Carlo integration was used to calculate the partition function in 26 .

${ }^{3}$ Recent proposals with lattice or non-lattice are found in $\left.10,28,11,12,8\right]$. See also 29$]$.
} 


\section{Basic Idea}

The idea for measuring the Witten index is made of two ingredients. One is for the regularization of the path integral measure and the other is for the regularization of the integral.

Let us start with the quantum mechanics. The standard way to obtain the path integral formulation from the operator formulation is discretizing the temporal direction and then inserting a complete set at each of the time slice:

$$
\left\langle q_{\mathrm{fin}}\left|e^{-i \hat{H} T}\right| q_{\mathrm{ini}}\right\rangle=\left(\prod_{k=1}^{N-1} \int_{-\infty}^{\infty} d q_{k}\right)\left\langle q_{\mathrm{fin}}\left|e^{-i a \hat{H}}\right| q_{N-1}\right\rangle\left\langle q_{N-1}\left|e^{-i a \hat{H}}\right| q_{N-2}\right\rangle \cdots\left\langle q_{1}\left|e^{-i a \hat{H}}\right| q_{\mathrm{ini}}\right\rangle,
$$

where $\left|q_{i}\right\rangle$ is a normalized state, $\hat{H}$ is the Hamiltonian 4 of the system, and the time difference of the initial state $\left|q_{\text {ini }}\right\rangle$ and the final state $\left|q_{\text {fin }}\right\rangle$ is $T=a N$. We regard this $a$ as the lattice spacing. Then the standard derivation 5 gives the path integral measure for a bosonic dimensionless lattice field $\phi^{\text {lat }}$ as follow:

$$
\int \mathcal{D} \phi^{\mathrm{lat}}=\prod_{k=0}^{N-1}\left[\left(\frac{1}{2 \pi}\right)^{\frac{1}{2}} \int_{-\infty}^{\infty} d \phi_{k}^{\mathrm{lat}}\right] .
$$

For dimensionless fermionic variables, we obtain

$$
\int \mathcal{D} \psi^{*} \mathcal{D} \psi=\prod_{k=0}^{N-1} \int d \psi_{k}^{*} d \psi_{k}
$$

In this way, we can determine the natural measure for the path integral.

In the lattice simulation, what we calculate is not the integration discussed so far. Instead, we calculate ensemble averages which give the ratios of the integrations. We have to establish a relation between these averages and the path integral regularized on the lattice.

In terms of the path integral, the expectation value is

$$
\langle A\rangle=\frac{\int \mathcal{D} \phi A e^{-S}}{\int \mathcal{D} \phi e^{-S}}
$$

where $S$ is the action of the system and we omit fermions for a while. The normalization, that is, the denominator is exactly the partition function which we are interested in. Since it is made of a ratio and does not depend on the normalization of the path integral, it seems impossible to measure the partition function with the correct normalization. However, let us consider the following quantity:

$$
\left\langle e^{+S} e^{-\frac{1}{2} \sum_{i} \mu^{2}\left(\phi_{i}^{\text {lat }}\right)^{2}}\right\rangle=\frac{\int \mathcal{D} \phi \exp \left[-\frac{1}{2} \sum_{i} \mu^{2}\left(\phi_{i}^{\text {lat }}\right)^{2}\right]}{\int \mathcal{D} \phi e^{-S}} \equiv \frac{C}{\int \mathcal{D} \phi e^{-S}},
$$

where $\mu$ is an arbitrary dimensionless parameter. With the measure defined in eq. (2.2), we obtain

$$
C=\int \mathcal{D} \phi e^{-\frac{1}{2} \sum_{i} \mu^{2} \phi_{i}^{2}}=\prod_{i=0}^{N-1}\left[\left(\frac{1}{2 \pi}\right)^{\frac{1}{2}} \int_{-\infty}^{\infty} d \phi_{i} e^{-\frac{1}{2} \sum_{i} \mu^{2} \phi_{i}^{2}}\right]=\mu^{-N} .
$$

\footnotetext{
${ }^{4}$ We explicitly denote the operators with hat $\left({ }^{\wedge}\right)$ in this section.

5 For the sake of completeness, we give a brief review of the derivation of path integral formulation in Appendix A
} 
Here, we have renamed the dimensionless lattice field $\phi_{i}^{\text {lat }} \rightarrow \phi_{i}$. For any real value of $\mu$, we can determine $C$ analytically which gives the overall normalization of the path integral. Note that $C$ is given just as an integration defined in (2.6), which is independent from the distribution of $\left\{\phi_{i}\right\}$. Especially, we never use Gaussian distributed quantities. The left hand side of eq. (2.5) is an observable in the simulation. Therefore, we can obtain the value of the partition function through the following expression:

$$
Z=\int \mathcal{D} \phi e^{-S}=\frac{C}{\left\langle e^{+S} e^{-\frac{1}{2} \sum_{i} \mu^{2} \phi_{i}^{2}}\right\rangle} .
$$

A comment on the choice of eq. (2.5) is in order here. We have chosen the Gaussian functional $e^{-\frac{1}{2} \sum_{i} \mu^{2} \phi_{i}^{2}}$ in eq. (2.5) for simplicity. As one can easily see, it can be replaced with any functional of $\phi$ as long as it gives a convergent and calculable value as in eq. (2.6).

Now let us introduce fermions. After integrating out the fermions, we obtain

$$
\int \mathcal{D} \psi^{*} \mathcal{D} \psi \mathcal{D} \phi e^{-S_{\mathrm{B}}-S_{\mathrm{F}}}=\int \mathcal{D} \phi \sigma[D] e^{-S^{\prime}}
$$

where $S_{\mathrm{B}}$ and $S_{\mathrm{F}}$ are the bosonic and fermionic part of the action, $S^{\prime}=S_{\mathrm{B}}-\ln |\operatorname{det} D|$ is an effective action with the kernel $D$ of the fermion bilinear, and $\sigma[D]$ is the sign (or complex phase) of det $D 6$ The configurations for the ensemble average are generated using the effective action $S^{\prime}$ ignoring the sign factor $\sigma[D]$. Let us denote the ensemble average over these configurations without the sign factor as $\langle\cdot\rangle_{0}$. The sign factor $\sigma[D]$ should be reweighted in the measurement afterwards:

$$
\langle A\rangle=\frac{\int \mathcal{D} \phi A \sigma[D] e^{-S^{\prime}}}{\int \mathcal{D} \phi \sigma[D] e^{-S^{\prime}}}=\frac{\langle\sigma[D] A\rangle_{0}}{\langle\sigma[D]\rangle_{0}}
$$

To determine the normalization of partition function, we also have to invert the effect of the sign factor in addition to the contribution from the effective action $e^{-S^{\prime}}$. The analogue of eq. (2.5) becomes $\left\langle\sigma[D]^{-1} e^{S^{\prime}-\frac{1}{2} \sum_{i} \mu^{2} \phi_{i}^{2}}\right\rangle=C / \int \mathcal{D} \phi \sigma[D] e^{-S^{\prime}}$, where the numerator gives again the integration (2.6). Now we obtain the expression for the Witten index $w$ :

$$
w=Z_{\mathrm{P}}=C \frac{\left\langle\sigma\left[D_{\mathrm{P}}\right]\right\rangle_{0, \mathrm{P}}}{\left\langle e^{S_{\mathrm{P}}^{\prime}-\frac{1}{2} \sum_{i} \mu^{2} \phi_{i}^{2}}\right\rangle_{0, \mathrm{P}}}
$$

provided all the fields are imposed the periodic boundary conditions as indicated by the suffix P.

Using pseudo fermion $\varphi$, we can rewrite eq. (2.10) as follow. Our definition of the measure gives exactly

$$
\int \mathcal{D} \psi^{*} \mathcal{D} \psi \exp \left[-\sum_{i, j} \psi_{i}^{*} D_{i j} \psi_{j}\right]=\int \mathcal{D} \varphi^{(1)} \mathcal{D} \varphi^{(2)} \sigma[D] \exp \left[-\sum_{i, j} \varphi_{i}^{*}\left(D^{\dagger} D\right)_{i j}^{-1 / 2} \varphi_{j}\right]=\operatorname{det} D,
$$

where $\varphi=\frac{1}{\sqrt{2}}\left(\varphi^{(1)}+i \varphi^{(2)}\right)$. Then, we obtain the expression for the index as

$$
w=Z_{\mathrm{P}}=C C_{\varphi} \frac{\left\langle\sigma\left[D_{\mathrm{P}}\right]\right\rangle_{0, \mathrm{P}}}{\left\langle e^{S_{\mathrm{P}}^{\prime \prime}-\frac{1}{2} \sum_{i} \mu^{2} \phi_{i}^{2}-\sum_{i} \mu_{\varphi}^{2} \varphi_{i}^{*} \varphi_{i}}\right\rangle_{0, \mathrm{P}}},
$$

\footnotetext{
${ }^{6}$ Depending on the number of the fermions, the determinant should be replaced by Pfaffian.
} 
where

$$
\begin{aligned}
S^{\prime \prime} & \equiv S_{\mathrm{B}}+\sum_{i, j} \varphi_{i}^{*}\left(D^{\dagger} D\right)_{i j}^{-1 / 2} \varphi_{j}, \\
C_{\varphi} & \equiv \int \mathcal{D} \varphi^{(1)} \mathcal{D} \varphi^{(2)} e^{-\sum_{i} \mu_{\varphi}^{2} \varphi_{i}^{*} \varphi_{i}} \cdot=\mu_{\varphi}^{-2 N} .
\end{aligned}
$$

It is interesting to see that there is a relation with the sign problem. Eq. (2.9) implies that the phase quenched average of the sign factor $\langle\sigma[D]\rangle_{0}$ is almost the partition function. If this average is close to 0 , we cannot obtain reliable expectation values numerically. This is the sign problem and in fact it occurs for supersymmetric quantum mechanics with spontaneously broken supersymmetry [19. Note that if the Witten index is vanishing, the partition function is vanishing so the expectation value becomes indefinite (or divergent) with periodic boundary conditions. Therefore it seems reasonable to regard (not) having the sign problem as an indication of the (non-)vanishing of the Witten index 7 However, no justification for this reasoning has been known, because no relation between the index and $\left\langle\sigma\left[D_{\mathrm{P}}\right]\right\rangle_{0, \mathrm{P}}$ has been known so far. Even if $\left\langle\sigma\left[D_{\mathrm{P}}\right]\right\rangle_{0, \mathrm{P}}$ is vanishing within the small error in the simulation, say $0.01 \pm 0.02$, without knowing the correct normalization, it could mean that the index is $0.001 \pm 0.002$ or $1.0 \pm 2.0$. Eq. (2.10) makes a connection between these two quantities and we can finally obtain the index from the sign factor.

One important disadvantage of the method is that it spoils the philosophy of the important sampling method due to the factor $e^{+S^{\prime}}$. Therefore, the choice of the value of $\mu$ is important. We have to chose it to have as large an overlap as possible between configurations and the operator we measure, namely $\exp \left[+S^{\prime}-\frac{1}{2} \sum_{i} \mu^{2} \phi_{i}\right]$. The only adjustable parameter to obtain larger overlap is $\mu$.

Finally, it is straightforward to extend our method to higher dimensional cases in principle. The efficiency of the simulation, however, could be far from practical because of the overlap problem we have just mentioned above.

\section{Analytical check}

The derivation of the path integral formulation from the operator formulation assumes the continuum limit. The lattice artifact may or may not spoil the previous argument. In this section, we discuss the effect of the exact supersymmetry on the lattice. We also give an explicit calculation of the index for the free case in supersymmetric quantum mechanics.

Let us suppose that the lattice action has an exact supersymmetry $Q$, like the one proposed in $[\underline{5}$. Then bosonic states and fermionic states make pairs even with finite lattice spacings, except for states with $Q^{\dagger} Q=0$. At this stage, the situation is exactly the same as in the continuum. The difference on the lattice is that we do not have $Q^{\dagger}$ as an exact symmetry on the lattice. However, supersymmetric vacuum must be annihilated by $Q$ and there is $Q$-exact Hamiltonian, so the conclusion does not change from the continuum case 8 . Even with finite lattice spacing, if the index is a non-zero integer supersymmetry is not broken. The advantage of exact invariance for the Witten index is also pointed out in 32 .

A more rigorous argument is the following. Using the fermion number operator $F$, the Witten index is written as

$$
w=\operatorname{Tr}\left((-1)^{F} e^{-\beta H}\right) .
$$

\footnotetext{
7 The fact that $\langle\sigma[D]\rangle_{0, P}$ has a close relation to the partition function and thus the Witten index has been pointed out in [19. See also 42 for a related numerical result.

${ }^{8}$ Except for the overall sign. See the discussion below.
} 
For simplicity, we assume that the spectrum is discrete and the index does not depend on $\beta(>0)$. For a $Q$-exact action $S=Q \Lambda$ with $Q^{2}=0$, it is natural to assume that the Hamiltonian $H$ in eq. (3.1) is also $Q$-exact:

$$
H=Q J_{0} .
$$

In fact, there is a natural way to define the $Q$-exact Hamiltonian [19].

Under the assumption of the $Q$-exactness of Hamiltonian, we can show that eq. (3.1) gives the index even with the finite lattice spacing. The Hamiltonian is complex in general in the $Q$-exact formulation so we have to introduce left- and right-eigen states, which satisfy

$$
H\left|L_{i}\right\rangle=E_{i}\left|L_{i}\right\rangle, \quad\left\langle R_{i}\right| H=\left\langle R_{i}\right| E_{i}, \quad\left\langle R_{i} \mid L_{j}\right\rangle=\delta_{i j} .
$$

The Witten index become

$$
w=\sum_{i}\left\langle R_{i}\left|e^{-\beta H}(-1)^{F}\right| L_{i}\right\rangle
$$

in this basis. Since $Q$ commutes with $H$ (note that $H=\left\{Q, J_{0}\right\}$ in the operator notation), $\left|L_{i}\right\rangle$ and $Q\left|L_{i}\right\rangle$ have the same eigen value $E_{i}$ for $H$. These two states have opposite statistics so they do not contribute to the index, except for the case $Q\left|L_{i}\right\rangle=0$. Similarly, $\left\langle R_{i}\right|$ and $\left\langle R_{i}\right| Q$ have the same eigen value and thus do not contribute except for the case $\left\langle R_{i}\right| Q=0$. Therefore, only $i$ such that $Q\left|L_{i}\right\rangle=0$ and $\left\langle R_{i}\right| Q=0$ contribute to the summation in eq. (3.4). These states have $E_{i}=0$, in fact:

$$
E_{i}=\left\langle R_{i}|H| L_{i}\right\rangle=\left\langle R_{i}\left|\left(Q J_{0}+J_{0} Q\right)\right| L_{i}\right\rangle=0 .
$$

Eventually, the index counts the number of the $E_{i}=0$ states with a weight factor $(-1)^{F}$, which is exactly the same as in the continuum case.

This result holds even at finite lattice spacing, because we have not assumed anything about the continuum limit 9 In particular, the index on the lattice must be an integer.

We can check the argument above and our measure for the path integral using a trivial example, i.e., the free supersymmetric quantum mechanics. The index should be 1 for the $Q$-exact lattice model.

The action of supersymmetric quantum mechanics with an exact $Q$ symmetry is

$$
\begin{aligned}
S= & -Q \sum_{k=0}^{N-1} \frac{1}{2} \bar{\psi}_{k}\left(F_{k}+\partial_{+} \phi_{k}+W^{\prime}\left(\phi_{k}\right)\right) \\
= & \sum_{k=0}^{N-1}\left[\frac{1}{2}\left(\phi_{k+1}-\phi_{k}\right)^{2}+\frac{1}{2} W^{\prime}\left(\phi_{k}\right)^{2}+\left(\phi_{k+1}-\phi_{k}\right) W^{\prime}\left(\phi_{k}\right)-\frac{1}{2} F_{k}^{2}\right. \\
& \left.\quad+\bar{\psi}_{k}\left(\psi_{k+1}-\psi_{k}\right)+W^{\prime \prime}\left(\phi_{k}\right) \bar{\psi}_{k} \psi_{k}\right] .
\end{aligned}
$$

Here, $\phi_{k}$ is a real scalar, $F_{k}$ is a bosonic auxiliary field, and $\psi_{k}$ is a complex fermion, $\partial_{+} \phi_{k}=\phi_{k+1}-\phi_{k}, W\left(\phi_{k}\right)$ is a potential and prime (') denotes the derivative w.r.t. $\phi_{k}$. All fields are rescaled to be dimensionless. $Q$-transformation, which is nilpotent, is:

$$
\begin{aligned}
Q \phi_{k} & =\psi_{k}, & Q \psi_{k} & =0, \\
Q \bar{\psi}_{k} & =F_{k}-\partial_{+} \phi_{k}-W^{\prime}\left(\phi_{k}\right), & & Q F_{k}=\partial_{+} \psi_{k}+W^{\prime \prime}\left(\phi_{k}\right) \psi_{k} .
\end{aligned}
$$

${ }^{9}$ Of course in order to guarantee that the continuum limit describes the target theory correctly, we have to check the restoration of full supersymmetry other than $Q$. 
In the following, we assume the free case:

$$
W\left(\phi_{k}\right)=\frac{1}{2} m \phi_{k}^{2},
$$

where $m=a m_{\text {phys }}$ is the dimensionless mass. The partition function with the periodic boundary conditions is

$$
\begin{aligned}
Z_{\mathrm{P}} & =\int_{\mathrm{P}} \mathcal{D} \phi \mathcal{D} F \mathcal{D} \bar{\psi} \mathcal{D} \psi e^{-S} \\
& =\prod_{i=1}^{N-1}\left(\int_{-\infty}^{\infty} \frac{d \phi_{i}}{\sqrt{2 \pi}} \int_{-\infty}^{\infty} \frac{d F_{i}}{\sqrt{2 \pi}} \int d \bar{\psi}_{i} d \psi_{i}\right) \exp \left[-\frac{1}{2} \sum_{i, j} \phi_{i} B_{i j} \phi_{j}+\frac{1}{2} \sum_{i} F_{i}^{2}-\sum_{i, j} \bar{\psi}_{i} D_{i j} \psi_{j}\right] \\
& =(\operatorname{det} B)^{-\frac{1}{2}} \operatorname{det} D
\end{aligned}
$$

where

$$
\begin{aligned}
& B_{i j}=(1-m)\left(2 \delta_{i j}-\delta_{i-1, j}-\delta_{i+1, j}\right)+m^{2} \delta_{i, j}, \\
& D_{i j}=\delta_{i+1, j}-\delta_{i, j}+m \delta_{i j},
\end{aligned}
$$

and $N$-periodicity is assumed for $B_{i j}$ and $D_{i j}$. In obtaining eq. (3.11), we made use of the replacement $F \rightarrow i F$ in the integrand to make the integration over the auxiliary field $F$ convergent. This gives unity with our measure and the contribution from the auxiliary field simply disappears. Alternatively, one can regard that we have moved to the on-shell formulation eliminating the auxiliary field, thus the integration should not contain $F$ from the beginning which gives the same result. It is straightforward to calculate the determinants $\operatorname{det} B$ and $\operatorname{det} D$. Using the momentum representation, and keeping all the overall normalization, we obtain

$$
\begin{aligned}
& \operatorname{det} B=\left(4-4 m+m^{2}\right) m^{2} \prod_{k=1}^{\frac{N}{2}-1}\left[(1-m) 4 \sin ^{2} \frac{\pi k}{N}+m^{2}\right]^{2}, \\
& \operatorname{det} D=(-2+m) m \prod_{k=1}^{\frac{N}{2}-1}\left[(1-m) 4 \sin ^{2} \frac{\pi k}{N}+m^{2}\right]
\end{aligned}
$$

for even $N$. Assuming $m<2$, which is the case if the lattice spacing is small enough, we obtain

$$
Z_{\mathrm{P}}=-1 \quad(N \text { : even }) .
$$

For odd $N$, we obtain

$$
\begin{aligned}
& \operatorname{det} B=m^{2} \prod_{k=1}^{\frac{N-1}{2}}\left[(1-m) 4 \sin ^{2} \frac{\pi k}{N}+m^{2}\right]^{2}, \\
& \operatorname{det} D=m \prod_{k=1}^{\frac{N-1}{2}}\left[(1-m) 4 \sin ^{2} \frac{\pi k}{N}+m^{2}\right],
\end{aligned}
$$

and thus

$$
Z_{\mathrm{P}}=1 \quad(N \text { : odd }) .
$$


Except for the minus sign for even $N$, we obtain the correct Witten index for the free case as expected.

What is the origin of the extra sign for even $N$ ? It comes from $\operatorname{det} D$. In the derivation of the path integral, we have

$$
\left\langle\psi_{k+1}\left|\hat{\psi}^{\dagger} \hat{\psi}\right| \psi_{l}\right\rangle=\psi_{k+1}^{*} \psi_{k}\left\langle\psi_{k+1} \mid \psi_{k}\right\rangle,
$$

that is, $\psi_{k+1}^{*}\left(=\bar{\psi}_{k+1}\right)$ is always combined with $\psi_{k}$. See eq. A.23) in Appendix A. A deviation from this combination would give the derivative as in the case in the kinetic term. For the action we have used here, however, a natural combination is $\bar{\psi}_{k} \psi_{k}$. The difference seems merely a lattice artifact but it gives a factor $\prod_{k=0}^{N-1} e^{\frac{2 \pi i}{N} k}=(-1)^{N+1}$ in the determinant, which explains the extra minus sign of $\operatorname{det} D$. On the other hand, the bosonic part does not have such an artifact because

$$
\left\langle q_{k+1}|\hat{q}| q_{k}\right\rangle=\left\langle q_{k+1}\left|q_{k}\right| q_{k}\right\rangle=\left\langle q_{k+1}\left|q_{k+1}\right| q_{k}\right\rangle,
$$

so it does not give any extra sign.

In the case of the naive action, the result is different. The lattice artifact survives and gives an extra contribution to the index. Let us start with the following action:

$$
S_{\text {naive }}=\sum_{i=0}^{N-1}\left[-\frac{1}{2} \phi_{i}\left(\phi_{i+1}+\phi_{i-1}-2 \phi_{i}\right)+\frac{1}{2} m^{2} \phi_{i}^{2}+\bar{\psi}_{i}\left(\psi_{i+1}-\psi_{i}\right)+m \bar{\psi}_{i} \psi_{i}\right] .
$$

Therefore, we have

$$
\begin{aligned}
B_{j k}^{\text {naive }} & =2 \delta_{j k}-\delta_{j, k+1}-\delta_{j+1, k}+m^{2} \delta_{j k}, \\
D_{j k}^{\text {naive }} & =\delta_{j+1, k}-\delta_{j k}+m \delta_{j k},
\end{aligned}
$$

which gives

$$
\frac{\operatorname{det} D^{\text {naive }}}{\left(\operatorname{det} B^{\text {naive }}\right)^{\frac{1}{2}}}= \begin{cases}\frac{-2+m}{\sqrt{4+m^{2}}} \prod_{k=1}^{\frac{N}{2}-1} \frac{4(1-m) \sin ^{2} \frac{k \pi}{N}+m^{2}}{4 \sin ^{2} \frac{k \pi}{N}+m^{2}} & (N \text { : even }) \\ \prod_{k=1}^{\frac{N-1}{2}-1} \frac{4(1-m) \sin ^{2} \frac{k \pi}{N}+m^{2}}{4 \sin ^{2} \frac{k \pi}{N}+m^{2}} & (N \text { : odd })\end{cases}
$$

The contribution from the factors in front of the product is $\mp 1$ in the continuum limit, where $m=a m_{\text {phys }} \rightarrow 0$. For even $N$, the extra minus sign appears again.

The problem is the inside of the product. Since there is no exact relation between bosonic modes and fermionic modes, there is no exact cancellation. Each term has a structure

$$
1-\mathcal{O}(m) \sim 1-\mathcal{O}(1 / N) .
$$

In the continuum limit, we obtain

$$
\frac{\operatorname{det} D^{\text {naive }}}{\left(\operatorname{det} B^{\text {naive }}\right)^{\frac{1}{2}}} \sim \prod_{k=1}^{N / 2}\left(1-\frac{\alpha}{N}\right) \sim e^{-\frac{\alpha}{2}}
$$

with a non-zero real number $\alpha$. It does not give the correct index even in the free case so we cannot use the naive action to measure the Witten index. 


\begin{tabular}{c|c} 
set & $\lambda_{2}^{\text {phys }}$ \\
\hline $2 \mathrm{a}$ & 0.5 \\
$2 \mathrm{~b}$ & 1.0 \\
$2 \mathrm{c}$ & 4.0
\end{tabular}

\begin{tabular}{c|c|c} 
set & $\lambda_{2}^{\text {phys }}$ & $\lambda_{3}^{\text {phys }}$ \\
\hline 3a & 4.0 & 4.0 \\
3b & 4.0 & 16.0 \\
3c & 4.0 & 32.0 \\
3d & 2.0 & 16.0
\end{tabular}

\begin{tabular}{c|c|c} 
set & $\lambda_{2}^{\text {phys }}$ & $\lambda_{4}^{\text {phys }}$ \\
\hline $4 \mathrm{a}$ & 1.0 & 1.0 \\
$4 \mathrm{~b}$ & 4.0 & 1.0 \\
$4 \mathrm{c}$ & 4.0 & 4.0
\end{tabular}

Table 1: Set labels for the free case $(n=2$, left), the $n=3$ interaction (middle) and the $n=4$ interaction (right). We set $L_{\text {phys }}=1$.

From the above argument, if the action has only $\mathcal{O}\left(a^{2}\right)$ or higher lattice artifact (even without having any part of exact supersymmetry), presumably we can use it to measure the Witten index. For dimension $d \geq 2$, the same argument allows only $\mathcal{O}\left(a^{d+1}\right)$ and higher lattice artifact, because the number of the product is $\sim N^{d}$. In the case of higher dimensions, however, we will need a fine tuning to obtain the supersymmetric continuum limit so the argument here is too naive.

\section{Numerical result}

In this section, we check our method using Monte Carlo simulation for supersymmetric quantum mechanics on the basis of the lattice formulation (3.7). We use the Hybrid Monte Carlo algorithm. See [19] for the implementation for this system 10. We fix the physical volume $L_{\text {phys }}=1$. The lattice spacing is $a=L_{\text {phys }} / N=1 / N$, where $N$ is the number of the lattice sites, and the bare coefficients $\lambda_{i}$ in the potential are scaled as $\lambda_{i}^{\text {phys }} N^{-i / 2}$. The parameters are summarized in table 1.

\subsection{Periodic case}

The very first check is the free case with periodic boundary conditions. There is only one bosonic vacuum and the index should be 1 . We list the result in table 2 and plot the index versus $\mu^{2}$ in fig. 1. We set $\mu^{2}=0.5,1.0,1.5,2.0,2.5,3.0$. The index from the simulation is in fact 1 within the error (at worst within 3 standard deviations) for odd $N$ and -1 for even $N$. This result exactly agrees with what we calculated in the previous section.

As table 2 2 shows, large $\frac{1}{2} m_{\text {phys }}=\lambda_{2}^{\text {phys }}$ (set 2c) requires less statistics, because the larger mass forces the scalar field to stay around the origin, which makes larger overlap with the quantity we measure. Note that large mass corresponds to low temperature measured in unit of $m_{\text {phys }}$, since it gives large $L_{\text {phys }} m_{\text {phys }}$.

Next case is the supersymmetric case with interactions. We use the $n=4$ potential

$$
W(\phi)=\lambda_{2} \phi^{2}+\lambda_{4} \phi^{4} .
$$

In this case, the index is known to be 1 for $\lambda_{2} \neq 0$. In table 3, we list the results for the index and in fig. 2 we plot the $\mu^{2}$ dependencies. It reproduces the correct index within the error (except for $\mu^{2}=0.5$ ). In particular, with a suitable choice of $\mu^{2}$ which gives small enough error, we can easily identify the integer. For some values of $\mu^{2}, 0.5$ for example, the error is rather large and we cannot determine the integer value. We expect, however, that if

\footnotetext{
${ }^{10}$ In addition, we change the time step $\delta \tau$ in the leap-frog precess as follows. In each trajectory we monitor the reversibility and if it is broken (it occurs if $\delta \tau$ is not small enough) we restart that trajectory using the same initial canonical momentum but smaller $\delta \tau$. Poor reversibilities are caused by very small eigen values of the Dirac operator.
} 


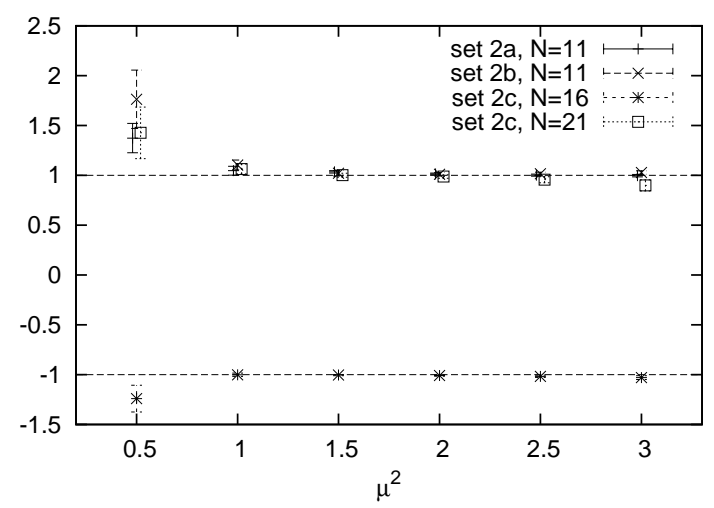

Figure 1: The index in the free case. It gives +1 for even $N$ and -1 for odd $N$ as discussed in the previous section.

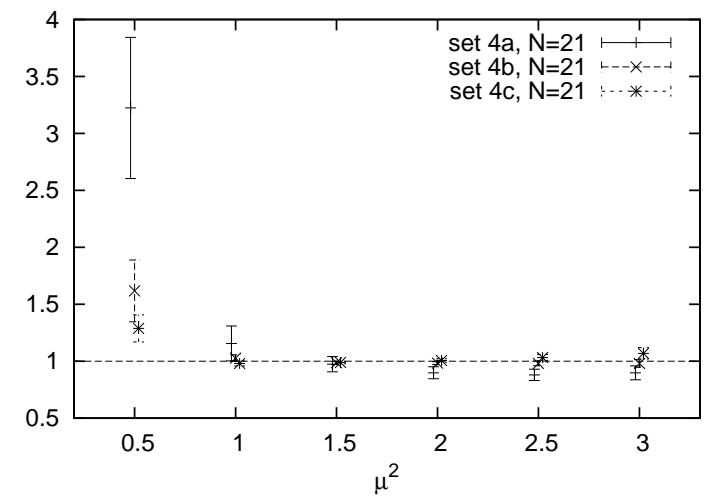

Figure 2: The index for the $n=4$ potential with various values of $\mu^{2}$. The dashed line represents the known result.

we used larger statistics it should converge to 1 . For the strong coupling (set 4c), we found that the configuration contained a non-negligible amount of artifact configurations at larger lattice spacing. We observed that for some bunch of configurations the artifact surface term becomes as much as 10 times larger (or more) than the other part of the action. This is the same phenomena reported in [43] in the 2-dimensional system. However, such artifact configurations disappear for smaller lattice spacing 11 We observed this phenomena only for the $n=4$ case.

In fig. 3. we plot the dependence on the lattice spacing $1 / N$, which shows that the index we measured is almost constant within the error against the lattice spacing.

Odd $n$ in the potential should give spontaneous breaking of supersymmetry. The Witten index is known to be 0 . We use $n=3$ potential:

$$
W(\phi)=\lambda_{2} \phi^{2}+\lambda_{3} \phi^{3} .
$$

We list the results of the simulation in table 4 and plot some of them in fig. 4 and 5 , against

\footnotetext{
11 The author thanks Hiroshi Suzuki for pointing out the disappearing.
} 


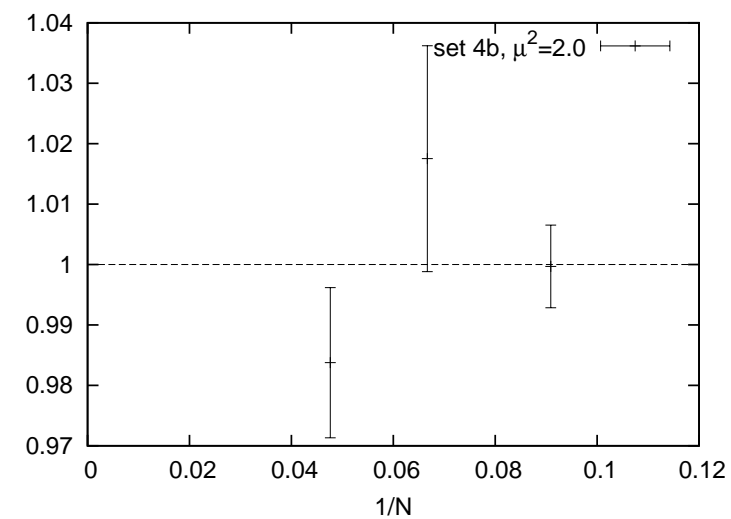

Figure 3: The index for the $n=4$ potential with different lattice spacings.

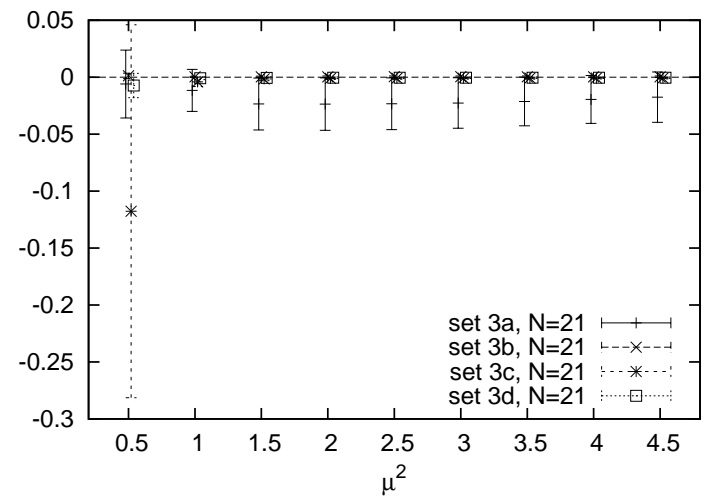

Figure 4: The index for the $n=3$ potential with various values of $\mu^{2}$. The dashed line represents the known result.

$\mu^{2}$ and $1 / N$, respectively. It gives in fact 0 as expected.

A comment on the statistics is in order. Since our measurement does not follow the philosophy of important sampling, it has an overlap problem in general. Therefore we need huge statistics. We use large enough number of independent configurations $N_{\text {confs }}$ to observe the behavior of the error $\sim N_{\text {confs }}^{-1 / 2}$ at least for some values of $\mu^{2}$ which give small error. See also the bottom of fig. 9 for a typical behavior of the error. For some values of $\mu^{2}$ which give large error (typically $\mu^{2}=0.5$ ), the statistics are not large enough, since the error did not behave as $\sim\left(N_{\text {confs }}\right)^{-1 / 2}$.

In the measurement, we used configurations every 10 trajectories for $n=2,4$ case and 100 trajectories for $n=3$. We used $O\left(10^{5}\right)-O\left(10^{6}\right)$ configurations which may or may not be independent. We estimated the error using the Jackknife method with several bin sizes. For $n=2,4$, there were almost no autocorrelations in the measurement so that the bin size we adopted is 1 for most sets of the parameter. For $n=3$ case, however, the autocorrelation becomes longer and the bin size we adopted is 20-250. This is because the sign $\sigma[D]$ does not change so frequently in the simulation. The other parts of the observable like $S^{\prime}$, which govern the convergence of error, have almost no auto correlations. Therefore, even if the 


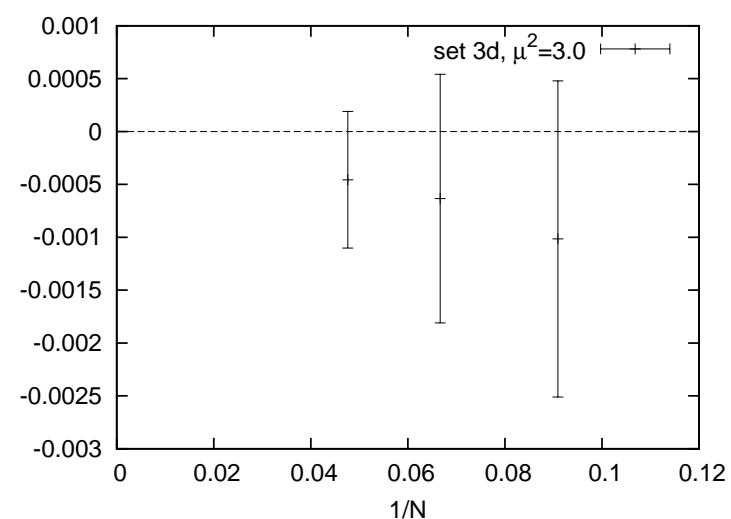

Figure 5: The index for the $n=3$ potential with different lattice spacings.

\begin{tabular}{c|c|r|l|l} 
set & $N$ & \multicolumn{1}{|c|}{ num. } & $\mu^{2}$ & \multicolumn{1}{|c}{ index } \\
\hline 2a & 11 & 499000 & 2.0 & $1.014(9)$ \\
\hline 2b & 11 & 499000 & 2.5 & $1.017(15)$ \\
\hline 2c & 11 & 499000 & 1.0 & $0.9997(18)$ \\
& 15 & 19900 & 1.5 & $0.990(7)$ \\
& 16 & 99800 & 1.5 & $-1.004(6)$ \\
& 21 & 499000 & 2.0 & $0.989(17)$
\end{tabular}

Table 2: The free $(n=2)$ case with periodic boundary conditions. We pick up $\mu^{2}$ which minimizes the error from $\mu^{2}=0.5,1.0,1.5, \ldots, 3.0$. The num. refers to the independent number of configurations after binning.

number of independent configurations is smaller than in the $n=4$ case, which is governed by $\sigma[D]$, the number of the configuration is large enough to give the error $\sim N_{\text {confs }}^{-1 / 2}$.

\subsection{Anti-Periodic case}

One may use anti-periodic boundary conditions for fermion in generating configurations. Using a reweighting method, we can afterwards obtain the desired index. In this case what we measure is

$$
w=C \frac{\left\langle e^{S_{\mathrm{AP}}^{\prime}} \sigma\left[D_{\mathrm{P}}\right] e^{-S_{\mathrm{P}}^{\prime}}\right\rangle_{0, \mathrm{AP}}}{\left\langle e^{S_{\mathrm{A}}^{\prime}-\frac{1}{2} \sum_{i} \mu^{2} \phi_{i}^{2}}\right\rangle_{0, \mathrm{AP}}},
$$

where we indicated the boundary condition, periodic (P) or anti-periodic (AP) explicitly in the suffix. Note the sign factor $\sigma$ must be calculated from the periodic Dirac operator $D_{\mathrm{P}}$.

In fig. 6 and 7, we plot some of the result against various values of $\mu^{2}$. The detailed results are given in table 5. It reproduces the desirable result as in the previous periodic case with suitably chosen $\mu^{2}$. Note that under the anti-periodic boundary conditions, supersymmetry is broken because we impose different conditions for the boson and the fermion. Even though, the $n=4$ case reproduces the correct index, although the temperature of the system is rather low for set $4 \mathrm{~b}$ thus the boundary condition may not give a large difference for the configurations. A rather non-trivial feature is found for the $n=3$ case. The average of $\sigma\left[D_{\mathrm{P}}\right]$ is not necessarily vanishing but the index is vanishing. For example, $\left\langle\sigma\left[D_{\mathrm{P}}\right]\right\rangle_{0, \mathrm{AP}}$ 


\begin{tabular}{c|c|r|r|l} 
set & $N$ & \multicolumn{1}{|c|}{ num. } & $\mu^{2}$ & \multicolumn{1}{|c}{ index } \\
\hline 4a & 11 & 199000 & 2.0 & $1.008(13)$ \\
& 15 & 499000 & 2.0 & $1.018(19)$ \\
& 21 & 999000 & 2.5 & $0.88(5)$ \\
\hline 4b & 11 & 99000 & 1.0 & $0.999(2)$ \\
& 15 & 49000 & 1.5 & $1.016(12)$ \\
& 21 & 999000 & 2.0 & $0.984(12)$ \\
\hline 4c & 11 & 99000 & 2.5 & $0.943(10)^{*}$ \\
& 15 & 99000 & 1.0 & $0.999(6)$ \\
& 21 & 499000 & 1.5 & $0.989(11)$
\end{tabular}

Table 3: The $n=4$ case with periodic boundary conditions. $\mu^{2}$ is chosen from $0.5,1.0, \ldots, 3.0$ to minimize the error. Configurations for the set $4 \mathrm{c}$ with $N=11$ contain artifact configurations so the value $\left(^{*}\right)$ is not reliable.

\begin{tabular}{c|l|r|r|l} 
set & $N$ & \multicolumn{1}{|c|}{ num. } & $\mu^{2}$ & \multicolumn{1}{|c}{ index } \\
\hline 3a & 11 & 1326 & 4.5 & $-0.008(14)$ \\
& 15 & 4995 & 2.0 & $0.002(5)$ \\
& 21 & 796 & 1.5 & $-0.024(23)$ \\
\hline 3b & 11 & 1237 & 4.5 & $0.005(4)$ \\
& 15 & 1326 & 3.0 & $-0.0018(21)$ \\
& 21 & 3118 & 4.0 & $0.0004(7)$ \\
\hline 3c & 11 & 4950 & 4.5 & $-0.001(3)$ \\
& 15 & 2450 & 3.5 & $0.003(4)$ \\
& 21 & 1237 & 3.0 & $-0.0009(8)$ \\
\hline 3d & 11 & 3326 & 4.5 & $-0.0010(14)$ \\
& 15 & 1243 & 3.0 & $-0.0006(12)$ \\
& 21 & 660 & 3.0 & $-0.0005(6)$
\end{tabular}

Table 4: The $n=3$ case with periodic boundary condition. $\mu^{2}$ is chosen from $0.5,1.0, \ldots, 4.5$ to minimize the error.

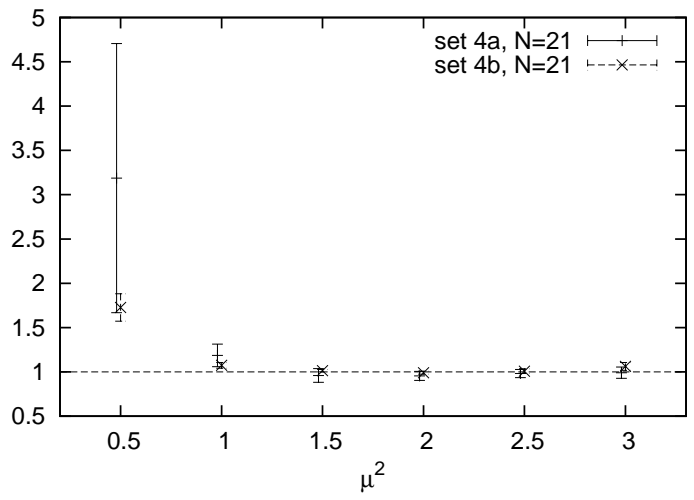

Figure 6: The index for the $n=4$ potential with various values of $\mu^{2}$. The configurations are generated with anti-periodic condition. The dashed line represents the known result. 


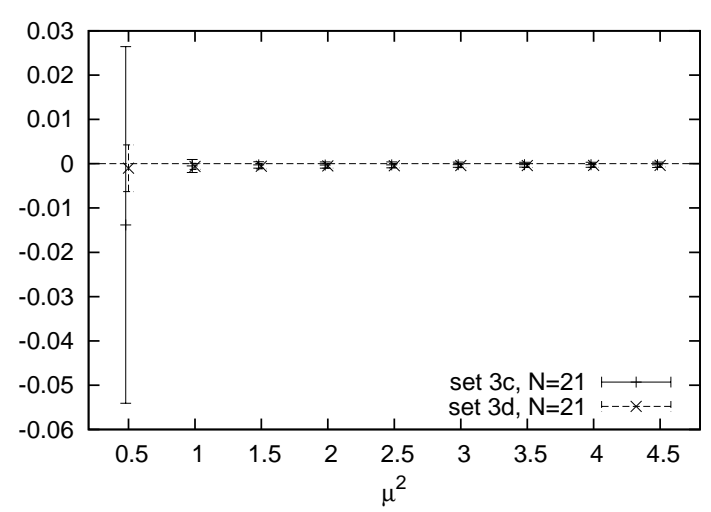

Figure 7: The index for the $n=3$ potential under various values of $\mu^{2}$. The configurations are generated with anti-periodic condition. The dashed line represents the known result.

\begin{tabular}{c|c|r|r|l} 
set & $N$ & \multicolumn{1}{|c|}{ num. } & $\mu^{2}$ & \multicolumn{1}{|c}{ index } \\
\hline 4a & 11 & 499000 & 2.0 & $0.999(8)$ \\
& 15 & 999000 & 2.0 & $0.996(13)$ \\
& 21 & 999000 & 2.5 & $0.98(5)$ \\
\hline 4b & 11 & 99000 & 1.0 & $0.9972(23)$ \\
& 15 & 99000 & 1.5 & $0.990(8)$ \\
& 21 & 499000 & 1.5 & $1.012(14)$
\end{tabular}

\begin{tabular}{c|c|r|r|l} 
set & $N$ & \multicolumn{1}{|c|}{ num. } & $\mu^{2}$ & \multicolumn{1}{|c}{ index } \\
\hline $3 \mathrm{c}$ & 11 & 1980 & 4.5 & $0.002(7)$ \\
& 15 & 1960 & 4.5 & $0.000(3)$ \\
& 21 & 6633 & 3.5 & $-0.0002(6)$ \\
\hline 3d & 11 & 1980 & 4.5 & $0.0007(27)$ \\
& 15 & 1237 & 2.5 & $0.000(1)$ \\
& 21 & 1980 & 4.5 & $-0.0004(4)$
\end{tabular}

Table 5: The index obtained from configurations with anti-periodic boundary conditions. $\mu^{2}$ is chosen from $0.5,1.0, \ldots, 3.0$ (set $4 \mathrm{a}, 4 \mathrm{~b}$ ) or $0.5,1.0, \ldots, 4.5$ (set $3 \mathrm{c}, 3 \mathrm{~d}$ ) to minimize the error.

for set $3 \mathrm{~d}$ with $N=21$ is $-0.018(7)$, which is slightly off from 0 , while the value of the index from $\mu^{2}=4.5$ is $-0.0004(4)$. The result shows that the reweighting in (4.3) works perfectly.

\subsection{The less important sampling method}

Since our method contradicts the philosophy of the important sampling, we can prepare the configurations using "less important" sampling. We replace the effective action $S^{\prime}$ in generating the configuration by $(1-r) S^{\prime}$, where $r$ is a real number. The ensemble average gives (assuming the large enough number of the statistics)

$$
\langle A\rangle_{r} \equiv \frac{\int \mathcal{D} \phi A[\phi] e^{-(1-r) S^{\prime}}}{\int \mathcal{D} \phi e^{-(1-r) S^{\prime}}} .
$$

In order to obtain the original expectation value, we have to use the reweighting method. For example,

$$
\langle A\rangle_{0}=\frac{\left\langle A e^{-r S^{\prime}}\right\rangle_{r}}{\left\langle e^{-r S^{\prime}}\right\rangle_{r}},
$$

and thus

$$
\langle A\rangle=\frac{\left\langle A \sigma[D] e^{-r S^{\prime}}\right\rangle_{r}}{\left\langle\sigma[D] e^{-r S^{\prime}}\right\rangle_{r}} .
$$




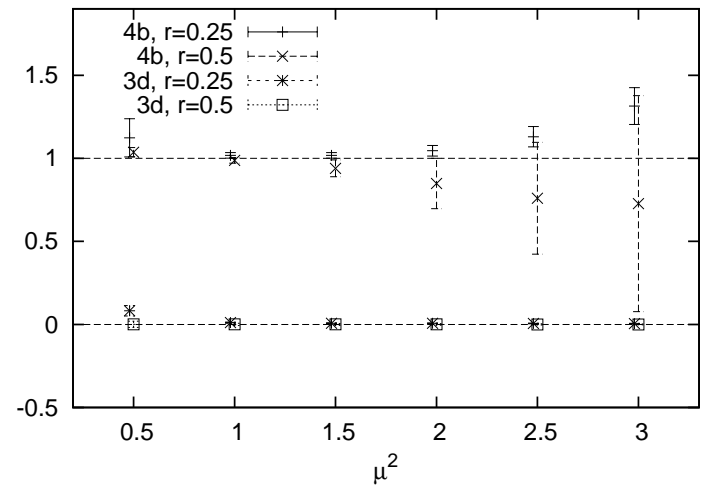

Figure 8: The index measured using the less important sampling for $N=21$. Set $4 \mathrm{~b}$ should give 1 and $3 \mathrm{~d}$ should give 0 .

The Witten index becomes

$$
w=C \frac{\left\langle\sigma\left[D_{\mathrm{P}}\right] e^{-r S_{\mathrm{P}}^{\prime}}\right\rangle_{r, \mathrm{P}}}{\left\langle e^{(1-r) S_{\mathrm{P}}^{\prime}-\frac{1}{2} \sum_{i} \mu^{2} \phi_{i}^{2}}\right\rangle_{r, \mathrm{P}}}
$$

for the configuration with the periodic boundary condition and

$$
w=C \frac{\left\langle\sigma\left[D_{\mathrm{P}}\right] e^{(1-r) S_{\mathrm{AP}}^{\prime}} e^{-S_{\mathrm{P}}^{\prime}}\right\rangle_{r, \mathrm{AP}}}{\left\langle e^{(1-r) S_{\mathrm{AP}}^{\prime}-\frac{1}{2} \sum_{i} \mu^{2} \phi_{i}^{2}}\right\rangle_{r, \mathrm{AP}}}
$$

for the anti-periodic conditions. Note that in gauge theories changing $r$ is nothing but changing the bare gauge coupling $\beta$.

Now the advantage of the less important sampling is clear. Let us set $r=\frac{1}{2}$ for simplicity and consider the periodic case. In eq. (2.10) the denominator has large fluctuations while the numerator has much smaller fluctuations. The behavior of the denominator requires large statistics and the unbalanced magnitude of the fluctuation with that of the numerator means a poor efficiency. In eq. (4.7), the denominator is the square root of that of eq. (2.10), so the order of the fluctuation becomes much smaller. In addition, the numerator has the same order of the fluctuation. In total, we expect much more efficient measurement.

In fig. 8, we show the result from the less important sampling method for periodicboundary conditions. With a suitable choice of $\mu^{2}$ which gives a small error, we can identify the correct index as expected. Table 6 summarizes the result based on the less important sampling method with $r=0.5$ and $r=0.25$. To see the advantage, we plot the behavior of the error against the number of configurations in fig. 9. picking up set 4b. Contrary to the expectation, the magnitudes of the error are not so different from one another for larger statistic 12 . But the less important sampling case gives a more stable behavior.

An interesting byproduct is that it makes it easier to flip the sign $\sigma\left[D_{\mathrm{P}}\right]$ for the $n=3$ case. The less important sampling method reduces the hight of the potential barrier between the $\sigma\left[D_{\mathrm{P}}\right]=1$ region and the -1 region. This effect gives less autocorrelation for the $n=3$ case compared with the $r=0$ cases before (with either periodic or anti-periodic conditions).

\footnotetext{
${ }^{12}$ If we had carefully optimized the value of $\mu^{2}$, the magnitude of the error for $r \neq 0$ might be significantly smaller than that for $r=0$.
} 


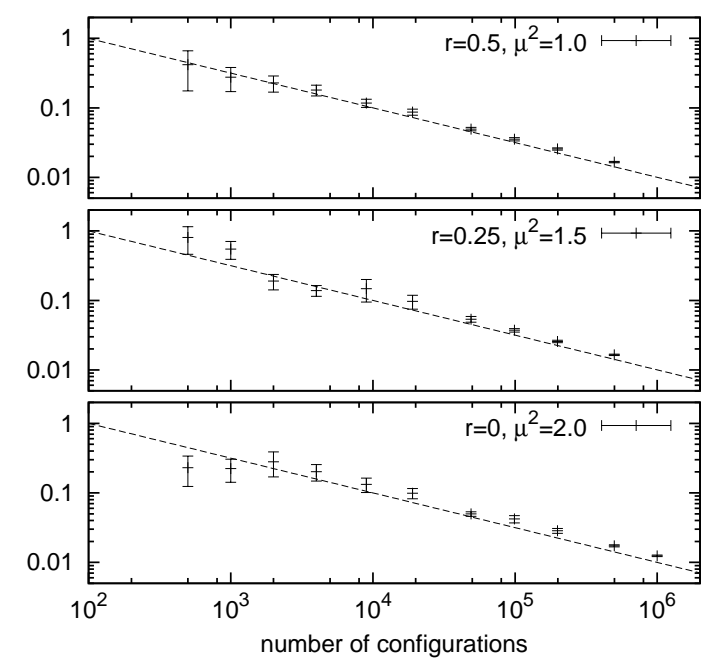

Figure 9: Behavior of the error versus number of independent configurations for different value of $r$. They are for set $4 \mathrm{~b}, N=21$ and $\mu^{2}$ with the smallest error. Dashed lines are $10 \times\left(N_{\text {confs }}\right)^{-1 / 2}$.

\begin{tabular}{l|l|r|r|l} 
set & $N$ & num. & $\mu^{2}$ & \multicolumn{1}{|c}{ index } \\
\hline 4b & 11 & 99000 & 1.0 & $1.0049(21)$ \\
& 15 & 99000 & 1.0 & $1.007(8)$ \\
& 21 & 499000 & 1.5 & $1.018(16)$ \\
\hline 3d & 11 & 450 & 3.0 & $0.017(6)$ \\
& 15 & 300 & 3.0 & $-0.008(6)$ \\
& 21 & 360 & 2.5 & $0.006(2)$
\end{tabular}

\begin{tabular}{l|l|r|l|l} 
set & $N$ & num. & $\mu^{2}$ & \multicolumn{1}{|c}{ index } \\
\hline 4b & 11 & 99000 & 1.0 & $0.995(3)$ \\
& 15 & 99000 & 1.0 & $1.010(11)$ \\
& 21 & 499000 & 1.0 & $0.987(17)$ \\
\hline 3d & 11 & 12475 & 2.5 & $-0.003(4)$ \\
& 15 & 4975 & 3.0 & $0.005(4)$ \\
& 21 & 9900 & 2.0 & $0.000(5)$
\end{tabular}

Table 6: Results from the less important sampling method with $r=0.25$ (left) and $r=0.5$ (right), under the periodic boundary conditions. $\mu^{2}$ is chosen from $0.5,1.0, \ldots, 3.0$ to minimize the error. 


\section{Conclusion and Discussion}

In this paper, we proposed a method for measuring the Witten index using a lattice simulation. The index is given by the average of the phase of the fermion determinant and we gave a suitable normalization to relate this average to the index. Our requirement for the lattice model is that it has at least one exact supersymmetry $Q$ with $Q^{2}=0$ and the action is $Q$-exact. We checked that the method reproduces the known Witten index for a simple supersymmetric quantum mechanics. The application to higher dimensional models is trivial in principle.

A disadvantage of the method is that it does not follow the philosophy of the important sampling. Therefore it requires high statistics. The situation would be worse the more degrees of freedom the model has. The efficiency for the higher dimensional model would be less practical. A less important sampling method may cure the situation to some extent.

In addition, having more fields means having more parameters to be tuned to achieve better efficiency. The supersymmetric quantum mechanics we numerically treated has only 1 parameter $\mu^{2}$, with our choice of Gaussian regularization functional. For the gauge theory, typically we have 3 such parameters; for scalar field, gauge field, and pseudo fermion field. One could even tune the regularization functional to have more parameters.

For the gauge theory, however, it is difficult to give a suitable gauge invariant regularization functional of the gauge fields. We also have to normalize the gauge volume. In the following, we discuss a possible treatment on the basis of the gauge fixing, though it contains some subtleties. In the lattice simulation, we can fix the gauge in the measurement setting as many link variables as possible to unity using the gauge transformation. The normalization constant $C$ should be obtained using only the remaining degrees of freedom. Note that in the constraint system the relevant degrees of freedom which contribute to the path integral are the remaining ones after the constraint is solved. Setting the link variables unity using the gauge transformation corresponds to solving the constraint. An important subtlety here, which may spoil this gauge fixing argument, is that it is not clear whether the gauge is completely fixed or not. Another subtlety is the regularization functional for which throughout in this paper we used a Gaussian functional. One can pick up the gauge field $A_{\mu}^{a}$ out of link variables and use the same Gaussian functional for $A_{\mu}^{a}$, but this relation contains some lattice artifacts. Therefore, it should give the correct normalization only after taking continuum limit. In this procedure the gauge fix is crucial since otherwise non gauge invariant Gaussian functional cannot give a non-trivial expectation value.

Our method can be presumably applied to a lattice model with a $Q$-closed term [16], which has an exact $Q$-invariance. In this case we cannot exclude contributions from $E>0$ states to the index. We have to take $L_{\text {phys }} \rightarrow \infty$ limit for the temporal direction.

\section{Acknowledgements}

The author thanks the Nishina Memorial Foundation for financial support. He thanks A. D'Adda for helpful discussions and reading the manuscript carefully. He also thanks to M. Billo, M. Hanada, R. Lineros, H. Matsufuru, H. Suzuki, M. Ünsal and U. Wenger for useful comments and discussions.

\section{A Derivation of the path integral}

We give a short review of the derivation of the path integral. 


\section{A.1 Bosons}

The bosonic Hamiltonian is

$$
\hat{H}(\hat{p}, \hat{q})=\frac{\hat{p}^{2}}{2 m_{\mathrm{QM}}}+V(\hat{q})
$$

where $m_{\mathrm{QM}}$ is a parameter with mass dimension 1 and $V(\hat{q})$ is a potential.

We calculate the amplitude

$$
\left\langle q_{N}(t=-i \tau=-i T) \mid q_{0}(t=-\tau=0)\right\rangle=\left\langle q_{N}\left|e^{-T \hat{H}}\right| q_{0}\right\rangle,
$$

where $\left|q_{0}\right\rangle$ and $\left|q_{N}\right\rangle$ are the initial and final state, respectively. The coordinate eigen state $|q\rangle$ is normalized as

$$
\left\langle q \mid q^{\prime}\right\rangle=\delta\left(q-q^{\prime}\right)
$$

and satisfies the completeness relation

$$
\int_{-\infty}^{\infty} d q|q\rangle\langle q|=1
$$

We divide the period $T$ into $N$ small periods:

$$
T=a N .
$$

Inserting $N-1$ completeness relations, we obtain

$$
\left\langle q_{N}\left|e^{-T \hat{H}}\right| q_{0}\right\rangle=\left(\prod_{k=1}^{N-1} \int_{-\infty}^{\infty} d q_{k}\right)\left\langle q_{N}\left|e^{-a \hat{H}}\right| q_{N-1}\right\rangle\left\langle q_{N-1}\left|e^{-a \hat{H}}\right| q_{N-2}\right\rangle \cdots\left\langle q_{1}\left|e^{-a \hat{H}}\right| q_{0}\right\rangle
$$

and assuming $a$ is small, we obtain

$$
\begin{aligned}
\left\langle q_{k+1}\left|e^{-a \hat{H}}\right| q_{k}\right\rangle & =\left\langle q_{k+1}|(1-a \hat{H})| q_{k}\right\rangle \\
& =\int_{-\infty}^{\infty} \frac{d p_{k+1}}{2 \pi} \exp \left[-a \frac{p_{k+1}^{2}}{2 m_{\mathrm{QM}}}-a V\left(\frac{q_{k+1}+q_{k}}{2}\right)+i p_{k+1}\left(q_{k+1}-q_{k}\right)\right] \\
& =\left(\frac{m_{\mathrm{QM}}}{2 \pi a}\right)^{\frac{1}{2}} \exp \left[-a\left(\frac{m_{\mathrm{QM}}}{2}\left(\frac{q_{k+1}-q_{k}}{2}\right)^{2}+V\left(\frac{q_{k+1}+q_{k}}{2}\right)\right)\right] .
\end{aligned}
$$

Here, we have assumed $\hat{H}$ is Weyl ordered. To calculate $\left\langle q_{k+1}|\hat{H}| q_{k}\right\rangle$, we have also used the momentum eigen state $|p\rangle$ which satisfies

$$
\langle p \mid q\rangle=\frac{1}{2 \pi} e^{-i p q}, \quad \quad \int_{-\infty}^{\infty} d p|p\rangle\langle p|=1
$$

In total, we obtain

$$
\begin{aligned}
\left\langle q_{N}\left|e^{-i T \hat{H}}\right| q_{0}\right\rangle= & \left(\frac{m_{\mathrm{QM}}}{2 \pi a}\right)^{\frac{N}{2}} \int_{-\infty}^{\infty}\left(\prod_{k=1}^{N-1} d q_{k}\right) \\
& \times \exp \left[-a \sum_{k=0}^{N-1}\left(\frac{m_{\mathrm{QM}}}{2}\left(\frac{q_{k+1}-q_{k}}{a}\right)^{2}+V\left(\frac{q_{k+1}+q_{k}}{2}\right)\right)\right] .
\end{aligned}
$$


In particular, the partition function becomes

$$
\begin{aligned}
\operatorname{Tr}\left(e^{-\hat{H} T}\right)= & \int_{-\infty}^{\infty} d q_{0}\left\langle q_{N}=q_{0}\left|e^{-\hat{H} T}\right| q_{0}\right\rangle \\
= & \prod_{k=0}^{N-1}\left[\left(\frac{m_{\mathrm{QM}}}{2 \pi a}\right)^{\frac{1}{2}} \int_{-\infty}^{\infty} d q_{k}\right] \\
& \times \exp \left[-a \sum_{k=0}^{N-1}\left(\frac{m_{\mathrm{QM}}}{2}\left(\frac{q_{k+1}-q_{k}}{a}\right)^{2}+V\left(\frac{q_{k+1}+q_{k}}{2}\right)\right)\right],
\end{aligned}
$$

where the periodic boundary condition $q_{N}=q_{0}$ is assumed in the exponent. Defining the 1-dimensional field $\phi_{k}=\sqrt{m_{\mathrm{QM}}} q_{k}$ (and suitable rescalings for parameters in the potential), we obtain

$$
\begin{aligned}
\operatorname{Tr}\left(e^{-\hat{H} T}\right)= & \prod_{k=0}^{N-1}\left[\left(\frac{1}{2 \pi a}\right)^{\frac{1}{2}} \int_{-\infty}^{\infty} d \phi_{k}\right] \\
& \times \exp \left[-a \sum_{k=0}^{N-1}\left(\frac{1}{2}\left(\frac{\phi_{k+1}-\phi_{k}}{a}\right)^{2}+V\left(\frac{\phi_{k+1}+\phi_{k}}{2}\right)\right)\right],
\end{aligned}
$$

where the first line provides the normalized measure. The exponent goes to the (minus) action in $a \rightarrow 0$ limit. Note that the above derivation gives one specific lattice action at finite $a$, which may or may not be useful for the lattice simulation.

For a dimensionless lattice field

$$
\phi_{k}^{\text {lat }}=a^{-\frac{1}{2}} \phi_{k}
$$

the measure becomes

$$
\int \mathcal{D} \phi^{\mathrm{lat}}=\prod_{k=0}^{N-1}\left[\left(\frac{1}{2 \pi}\right)^{\frac{1}{2}} \int_{-\infty}^{\infty} d \phi_{k}^{\mathrm{lat}}\right]
$$

\section{A.2 Fermions}

We use the following convention for the Grassmann integration:

$$
\int d \psi^{*} d \psi \psi \psi^{*}=+1
$$

The creation and annihilation operators $\hat{\psi}$ and $\hat{\psi}^{\dagger}$ satisfies the following anti-commutators:

$$
\left\{\hat{\psi}, \hat{\psi}^{\dagger}\right\}=1, \quad\{\hat{\psi}, \hat{\psi}\}=\left\{\hat{\psi}^{\dagger}, \hat{\psi}^{\dagger}\right\}=0 .
$$

The Hilbert space is 2-dimensional and spanned by $|0\rangle$ and $|1\rangle$ as usual:

$$
\begin{aligned}
\hat{\psi}|0\rangle & =0, & \hat{\psi}^{\dagger}|0\rangle & =|1\rangle, \\
\langle 0 \mid 0\rangle & =\langle 1 \mid 1\rangle=1, & \langle 0 \mid 1\rangle & =0 .
\end{aligned}
$$

The Hamiltonian for fermions is

$$
\hat{H}\left(\hat{\psi}^{\dagger}, \hat{\psi}\right)=\hat{\psi}^{\dagger} M \hat{\psi}
$$


It is convenient to introduce the following coherent state:

$$
|\psi\rangle \equiv e^{-\psi \hat{\psi}^{\dagger}}|0\rangle=|0\rangle-\psi|1\rangle,
$$

where $\psi$ (without ${ }^{\wedge}$ ) is a Grassmann odd c-number. This state satisfies

$$
\hat{\psi}|\psi\rangle=\psi|\psi\rangle \quad(=\psi|0\rangle) .
$$

The orthogonality and the completeness relations are

$$
\begin{aligned}
\left\langle\psi \mid \psi^{\prime}\right\rangle & =1+\psi^{*} \psi^{\prime}=e^{\psi^{*} \psi^{\prime}} \\
\int d \psi^{*} d \psi|\psi\rangle e^{-\psi^{*} \psi}\langle\psi| & =|0\rangle\langle 0|+| 1\rangle\langle 1|=1
\end{aligned}
$$

Inserting $N-1$ complete sets and using

$$
\begin{aligned}
\left\langle\psi_{k+1}\left|e^{-a \hat{H}}\right| \psi_{k}\right\rangle & =\left\langle\psi_{k+1}\left|\left(1-a H\left(\psi_{k+1}^{*}, \psi_{k}\right)\right)\right| \psi_{k}\right\rangle \\
& =\exp \left[-a H\left(\psi_{k+1}^{*}, \psi_{k}\right)+\psi_{k+1}^{*} \psi_{k}\right],
\end{aligned}
$$

we obtain

$$
\begin{array}{r}
\left\langle\psi_{N}\left|e^{-\hat{H} T}\right| \psi_{0}\right\rangle=\left(\prod_{k=1}^{N-1} \int d \psi_{k}^{*} d \psi_{k}\right) \exp \left[-a \sum_{k=1}^{N-1}\left\{H\left(\psi_{k+1}^{*}, \psi_{k}\right)+\frac{1}{a} \psi_{k}^{*}\left(\psi_{k}-\psi_{k-1}\right)\right\}\right. \\
\left.+\psi_{N}^{*} \psi_{N-1}-a H\left(\psi_{1}^{*}, \psi_{0}\right)\right] .
\end{array}
$$

The trace of an operator $\hat{A}$ in the coherent representation is

$$
\operatorname{Tr}(\hat{A})=-\int d \psi^{*} d \psi e^{\psi^{*} \psi}\langle\psi|\hat{A}| \psi\rangle=\langle 0|\hat{A}| 0\rangle+\langle 1|\hat{A}| 1\rangle .
$$

Then we obtain

$$
\begin{aligned}
& \operatorname{Tr}\left(e^{-\hat{H} T}\right)=-\int d \psi_{0}^{*} d \psi_{0} e^{\psi_{0}^{*} \psi_{0}}\left\langle\psi_{0}\left|e^{-\hat{H} T}\right| \psi_{0}\right\rangle \\
&=-\left(\prod_{k=0}^{N-1} \int d \psi_{k}^{*} d \psi_{k}\right) \exp \left[-a \sum_{k=1}^{N-1}\left\{H\left(\psi_{k}^{*}, \psi_{k-1}\right)+\frac{1}{a} \psi_{k}^{*}\left(\psi_{k}-\psi_{k-1}\right)\right\}\right. \\
&\left.\quad-a H\left(\psi_{0}^{*}, \psi_{N-1}\right)+\psi_{0}^{*} \psi_{N-1}+\psi_{0}^{*} \psi_{0}\right]
\end{aligned}
$$

Replacing $\psi_{0}^{*} \rightarrow-\psi_{0}^{*}$ and imposing the anti-periodic condition $\psi_{N} \equiv-\psi_{0}$ and $\psi_{N}^{*} \equiv-\psi_{0}^{*}$, we obtain

$$
\operatorname{Tr}\left(e^{-\hat{H} T}\right)=\left(\prod_{k=0}^{N-1} \int d \psi_{k}^{*} d \psi_{k}\right) \exp \left[-a \sum_{k=0}^{N-1}\left\{H\left(\psi_{k+1}^{*}, \psi_{k}\right)+\frac{1}{a} \psi_{k+1}^{*}\left(\psi_{k+1}-\psi_{k}\right)\right\}\right] .
$$

In the continuum limit $a \rightarrow 0$, the exponent gives the Euclidean action with anti-periodic boundary conditions.

To conclude, the measure for the fermion field is

$$
\int \mathcal{D} \psi^{*} \mathcal{D} \psi=\prod_{k=0}^{N-1} \int d \psi_{k}^{*} d \psi_{k}
$$




\section{References}

[1] S. Catterall, D. B. Kaplan and M. Unsal, Phys. Rept. 484 (2009) 71 arXiv:0903.4881 [hep-lat]].

[2] D. B. Kaplan, E. Katz and M. Unsal, JHEP 0305 (2003) 037 arXiv:hep-lat/0206019.

[3] S. Catterall and S. Karamov, Phys. Rev. D 65 (2002) 094501 arXiv:hep-lat/0108024.

[4] I. Kanamori and H. Suzuki, Nucl. Phys. B 811 (2009) 420 arXiv:0809.2856 [hep-lat]].

[5] F. Sugino, JHEP 0403 (2004) 067 arXiv:hep-lat/0401017.

[6] A. D'Adda, I. Kanamori, N. Kawamoto and K. Nagata, Nucl. Phys. B 707 (2005) 100 arXiv:hep-lat/0406029.

A. D'Adda, I. Kanamori, N. Kawamoto and K. Nagata, Phys. Lett. B 633 (2006) 645 arXiv:hep-lat/0507029.

[7] A. D'Adda, N. Kawamoto and J. Saito, Phys. Rev. D 81 (2010) 065001 arXiv:0907.4137 [hep-th]].

[8] A. D'Adda, A. Feo, I. Kanamori, N. Kawamoto and J. Saito, arXiv:1006.2046 [hep-lat].

[9] P. H. Damgaard and S. Matsuura, JHEP 0709 (2007) 097 arXiv:0708.4129 [hep-lat]].

[10] M. Hanada, J. Nishimura and S. Takeuchi, Phys. Rev. Lett. 99 (2007) 161602 arXiv:0706.1647 [hep-lat]].

[11] D. Kadoh and H. Suzuki, Phys. Lett. B 684 (2010) 167 [arXiv:0909.3686 [hep-th]].

[12] G. Bergner, JHEP 1001 (2010) 024 [arXiv:0909.4791 [hep-lat]].

[13] T. Ishii, G. Ishiki, S. Shimasaki and A. Tsuchiya, Phys. Rev. D 78 (2008) 106001 arXiv:0807.2352 [hep-th]].

G. Ishiki, S. W. Kim, J. Nishimura and A. Tsuchiya, JHEP 0909 (2009) 029 arXiv:0907.1488 [hep-th]].

[14] D. Kadoh, F. Sugino and H. Suzuki, Nucl. Phys. B 820 (2009) 99 arXiv:0903.5398 [hep-lat]].

[15] D. Kadoh and H. Suzuki, Phys. Lett. B 682 (2010) 466 [arXiv:0908.2274 [hep-lat]].

[16] M. Hanada, S. Matsuura and F. Sugino, arXiv:1004.5513 [hep-lat].

[17] H. Kawai and Y. Kikukawa, arXiv:1005.4671 [hep-lat].

[18] M. Beccaria, M. Campostrini and A. Feo, Phys. Rev. D 69 (2004) 095010 arXiv:hep-lat/0402007.

[19] I. Kanamori, H. Suzuki and F. Sugino, Phys. Rev. D 77 (2008) 091502 arXiv:0711.2099 [hep-lat]].

I. Kanamori, F. Sugino and H. Suzuki, Prog. Theor. Phys. 119 (2008) 797 arXiv:0711.2132 [hep-lat]]. 
[20] K. N. Anagnostopoulos, M. Hanada, J. Nishimura and S. Takeuchi, Phys. Rev. Lett. 100 (2008) 021601 arXiv:0707.4454 [hep-th]].

M. Hanada, A. Miwa, J. Nishimura and S. Takeuchi, Phys. Rev. Lett. 102 (2009) 181602 arXiv:0811.2081 [hep-th]].

M. Hanada, Y. Hyakutake, J. Nishimura and S. Takeuchi, Phys. Rev. Lett. 102 (2009) 191602 arXiv:0811.3102 [hep-th]].

M. Hanada, J. Nishimura, Y. Sekino and T. Yoneya, Phys. Rev. Lett. 104 (2010) 151601 arXiv:0911.1623 [hep-th]].

[21] S. Catterall and T. Wiseman, Phys. Rev. D 78 (2008) 041502 arXiv:0803.4273 [hep-th]].

S. Catterall and T. Wiseman, JHEP 0712 (2007) 104 arXiv:0706.3518 [hep-lat]].

[22] M. Hanada and I. Kanamori, Phys. Rev. D 80 (2009) 065014 [arXiv:0907.4966 [hep-lat]].

[23] E. Witten, Nucl. Phys. B 202 (1982) 253.

[24] S. Cecotti and L. Girardello, Phys. Lett. B 110 (1982) 39.

[25] K. Fujikawa, Z. Phys. C 15 (1982) 275.

[26] W. Krauth, H. Nicolai and M. Staudacher, Phys. Lett. B 431 (1998) 31 arXiv:hep-th/9803117.

[27] E. Witten, Nucl. Phys. B 188 (1981) 513.

[28] S. Arianos, A. D'Adda, A. Feo, N. Kawamoto and J. Saito, Int. J. Mod. Phys. A 24 (2009) 4737 arXiv:0806.0686 [hep-lat]].

[29] G. Bergner, T. Kaestner, S. Uhlmann and A. Wipf, Annals Phys. 323 (2008) 946 arXiv:0705.2212 [hep-lat]].

[30] S. Catterall and E. Gregory, Phys. Lett. B 487 (2000) 349 arXiv:hep-lat/0006013.

[31] S. Catterall, JHEP 0305 (2003) 038 arXiv:hep-lat/0301028.

[32] J. Giedt, R. Koniuk, E. Poppitz and T. Yavin, JHEP 0412 (2004) 033 arXiv:hep-lat/0410041.

[33] M. Beccaria, G. Curci and E. D'Ambrosio, Phys. Rev. D 58 (1998) 065009 arXiv:hep-lat/9804010.

[34] T. Banks, W. Fischler, S. H. Shenker and L. Susskind, Phys. Rev. D 55 (1997) 5112 arXiv:hep-th/9610043.

[35] P. Yi, Nucl. Phys. B 505 (1997) 307 arXiv:hep-th/9704098.

[36] S. Sethi and M. Stern, Commun. Math. Phys. 194 (1998) 675 arXiv:hep-th/9705046.

[37] G. W. Moore, N. Nekrasov and S. Shatashvili, Commun. Math. Phys. 209 (2000) 77 arXiv:hep-th/9803265].

[38] J. Wosiek, Nucl. Phys. B 644 (2002) 85 arXiv:hep-th/0203116].

[39] M. Campostrini and J. Wosiek, Phys. Lett. B 550 (2002) 121 arXiv:hep-th/0209140. 
[40] M. Campostrini and J. Wosiek, Nucl. Phys. B 703 (2004) 454 arXiv:hep-th/0407021.

[41] P. Korcyl, arXiv:0912.5265 [hep-th].

[42] S. Catterall, JHEP 0901 (2009) 040 [arXiv:0811.1203 [hep-lat]].

[43] T. Kastner, G. Bergner, S. Uhlmann, A. Wipf and C. Wozar, Phys. Rev. D 78 (2008) 095001 arXiv:0807.1905 [hep-lat]]. 